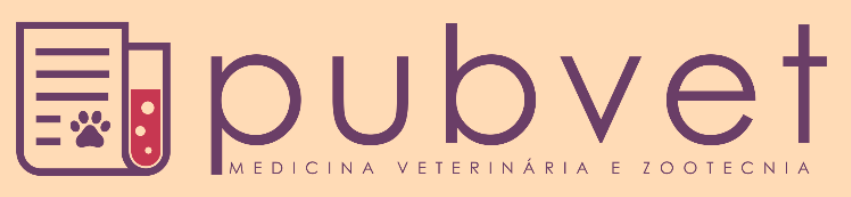

https://doi.org/10.31533/pubvet.v13n8a384.1-5

\title{
Efeito da progesterona exógena injetável em protocolos de sincronização em ovinos
}

\author{
Isadora Bretanha $^{1^{*}}$, Caio Victor Damasceno Carvalho $^{2} \bullet$, Misael Caldas Nascimento ${ }^{3} \bullet$ \\ ${ }^{I}$ Discente da União Metropolitana de Educação e Cultura, Lauro de Freitas - BA Brasil \\ ${ }^{2}$ Professor Doutor da Universidade Federal do Oeste da Bahia, Barreiras-BA Brasil \\ ${ }^{3}$ Professor Doutor da União Metropolitana de Educação e Cultura, Lauro de Freitas - BA Brasil- \\ *Autor para correspondência, E-mail: dorabreta.vet@gmail.com
}

Resumo. O objetivo desse trabalho foi demonstrar os efeitos da progesterona exógena injetável em protocolos de indução e sincronização de ciclo estral em ovinos. A produção desses animais mostrou-se crescente no estado da Bahia, impulsionando a necessidade de novas técnicas que aplicadas ao manejo reprodutivo aumentando a taxa de produtividade dos rebanhos. Dessa forma, foram conduzidos dois protocolos hormonais de curta duração em 17 matrizes da raça Santa Inês. Dois grupos foram submetidos à associação de Progesterona Injetável, Gonadotrofina Coriônica Equina (eCG) e Cloprostenol Sódico como precursor de Prostaglandina $2 \alpha$ (PGF2 $\alpha$ ), diferindo nas doses e intervalo de aplicação. Os resultados obtidos em relação a apresentação de cio não foram significativos, já os índices de parição sofreram influência negativa após o protocolo.

Palavras chave: estro, ovelhas, progestágenos, reprodução

\section{Effect of injectable exogenous progesterone on synchronization protocols in sheep}

\begin{abstract}
The objective of this work was to demonstrate the effects of exogenous injectable progesterone on protocols of induction and estrus cycle synchronization in sheep. The production of these animals has shown to be increasing in the state of Bahia, impelling the need for new techniques applied to reproductive management to raise the rate of productivity of the herds. Thus, two hormonal protocols of short duration were conducted in 17 Santa Inês breeders. Two groups were submitted to combination of Progesterone Injection, Equine Chorionic Gonadotrophin $(\mathrm{eCG})$ and Clorprostenol Sodium as precursor of Prostaglandin $2 \alpha(\mathrm{PGF} 2 \alpha)$, differing in doses and application interval. The results obtained in relation to estrus presentation were not significant, since parity indexes had a negative influence after the protocol.
\end{abstract}

Keywords: estrus, ewes, progestins, reproduction

\section{Efecto de la progesterona exógena inyectable en protocolos de sincronización en ovinos}

Resumen. El objetivo de este trabajo fue demostrar los efectos de la progesterona exógena inyectable en protocolos de inducción y sincronización de ciclo estral en ovinos. La producción de esta especie ha aumentado en el estado de Bahia, impulsando la necesidad de nuevas técnicas que aplicadas al manejo reproductivo eleven la tasa de productividad de los rebaños. De esta forma, se realizaron dos protocolos hormonales de corta duración en 17 matrices de la raza Santa Inés. Dos grupos fueron sometidos a la asociación de 
Progesterona Inyectable, Gonadotrofina Coriónica Equina (eCG) y Cloprostenol Sódico como precursor de Prostaglandina $2 \alpha$ (PGF2 $\alpha$ ), diferenciándose en las dosis e intervalo de aplicación. Los resultados obtenidos con relación a la presentación de celo no fueron significativos, en relación con los índices de parición, estos sufrieron influencia negativa después del realizar el protocolo de inducción.

Palabras clave: Estro, ovejas, progestágeno, reproducción

\section{Introdução}

A produção de ovinos é crescente no estado da Bahia (ANUALPEC, 2018) e para que se alcance maior produtividade são aplicados, manejos reprodutivos que visam elevar as taxas de fertilidade. Sendo assim, o objetivo desse trabalho é avaliar os efeitos da progesterona injetável em protocolos de estimulação de cio em ovinos. Na realização de técnicas para indução e sincronização estral faz-se o uso de drogas como progestágenos, eCG (gonadotrofina coriônica equina) e prostaglandinas (UribeVelásquez et al., 2010).

A progesterona ( $\mathrm{P} 4)$ é um hormônio esteroide, que possui função reguladora no sistema reprodutivo das fêmeas sendo efetivo em concentrações baixas, por isso, são sintetizadas em quantidades relativamente pequenas nos ovários e placenta, interferindo de diferentes formas no ciclo estral, dependendo da fase em que se encontra (Lehninger, 2006). Durante a foliculogênese, a P4 atua como coadjuvante nos períodos de recrutamento, seleção e desenvolvimento dos folículos. A elevação dos níveis de FSH estimula o recrutamento e emergência folicular (Sousaet al., 2015). Além disso, esse hormônio desempenha função no crescimento, impulsionando a formação do antro, estimulação das células da granulosa à produção de secreção e proporcionando disponibilidade de mais receptores para o Hormônio Luteinizante (LH). Por sua vez, o LH tem como funções, ação conjunta ao FSH na maturação folicular, promoção da liberação do oócito e estimulação da luteinização. O pico de Hormônio Luteinizante é possível devido ao mecanismo de aumento dos pulsos de sua liberação, que é baseado na retroalimentação positiva regida pelo Estrógeno (E2) (Reece \& PenteadoJúnior, 1996). É atribuída à influência estrogênica o desencadeamento do comportamento de cio apresentado pelas fêmeas no período de estro, desde que associado a baixos níveis de P4. Assim, induzido pela sinergia entre esses dois hormônios, torna-se possível à ocorrência de oocitação (Uribe-Velásquez et al., 2008)Em seguida, dá-se início a fase luteal, que é caracterizada pela presença de um Corpo Lúteo (CL) (Colazo \& Mapletoft, 2017). No caso de não ocorrência da fecundação do oocito liberado, o CL permanece em atividade por 14 a 15 dias, sendo sua lise proporcionada por ação da prostaglandina $2 \alpha$ (PGF2 $\alpha$ ). Havendo fecundação, as células luteínicas são responsáveis pela produção de elevados níveis de progesterona circulante, objetivando a manutenção da gestação por meio de efeitos antiestrogênicos.

Os protocolos mais utilizados envolvem dispositivos intravaginais com progesterona ou outros progestágenos, juntamente com o uso eCG (Souza, 2013). Contudo, a utilização de implantes possui desvantagens como desenvolvimento de vaginite, sendo as alterações hormonais no sistema reprodutivo e carreamento de bactérias para o ambiente vaginal, fatores determinantes para o desenvolvimento da afecção. $\mathrm{O}$ custo alto desses procedimentos também pode ser um fator limitante para sua implantação (Castilho et al., 2013). Considerando que o preço da unidade de implante de progesterona alcança o valor de $\mathrm{R} \$ 22,00$, enquanto na aplicação de $1,0 \mathrm{ml}$ de progesterona injetável seria de $\mathrm{R} \$ 1,00$, a utilização da segunda opção reduziria consideravelmente os custos na implantação de protocolos em rebanhos ovinos. Além disso, o manejo de implantes requer mais tempo de mão de obra empregada, pois gasta-se tempo na colocação e retirada dos pessários, enquanto a aplicação de hormônio na forma injetável necessita da mão de obra apenas em uma aplicação.

A escolha da via de administração foi baseada na necessidade de novas formas de aplicação da progesterona, possibilitando a redução de transtornos advindos da introdução de implantes subcutâneos ou intravaginais. A via parenteral foi à opção eleita mesmo com o risco de necessidade de repetição do tratamento, devido à baixa precisão de seu nível de biodisponibilidade.

Dessa forma, a constante necessidade de aperfeiçoamento e criação de técnicas de sincronização hormonal, impulsionou esse projeto afim de proporcionar maior rentabilidade em diversos sistemas de produção da ovinocultura. 


\section{Material e métodos}

Os protocolos de sincronizações foram conduzidos em uma propriedade rural selecionadora de

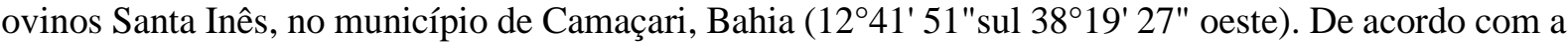
classificação de Köppen \& Geiger (1928) se insere no clima de regiões tropicais com estação de chuvas no inverno. Os animais foram mantidos em pastejo rotacionado com suplementação. Durante o período da manhã ocorreu o pastejo em Bachiaria humidicola, Panicum maximum CV. Massai e Digitaria pentzii Stentconhecida como Capim-Pangolão. A suplementação aconteceu no período da tarde e foi baseada no oferecimento de Pennisetum purpureum (Capim Napier) juntamente com ração possuindo níveis de Umidade máxima de 13\%, Proteína Bruta de 16\%, NNP- Equivalente Proteína (máx) de 2,2\%, Extrato Etéreo (máx) de 3\%, Matéria mineral (máx) de 12\%, Vitamina A 12.500 UI/ kg, Vitamina D3 de 2000 UI /kg, Vitamina E 17,00 UI/ kg e Monensina Sódica a 0,002 \%. A fonte de mineralização e água foram oferecidas ad libitum. O sal mineral oferecido continha22,5\% de Cálcio; 7,5\% de Fósforo; 0,075\% de Flúor; 9\% de sódio, $1 \%$ de enxofre; 0,5\% de Magnésio; 0,04 \% de ferro; 0,004\% de Iodo; $0,1 \%$ de Manganês; $0,0024 \%$ de Selênio, $0,002 \%$ de Cobalto e 0,3\% de Zinco, Vitamina A (min) $310.000 \mathrm{UI} / \mathrm{kg}$, Vitamina D3(min) $50.000 \mathrm{UI} / \mathrm{kg}$ e Vitamina E 435,00 UI/kg.

O manejo sanitário na propriedade consistiu em medidas de prevenção e tratamento de doenças que acometem rebanhos ovinos. A limpeza das instalações foi feita uma vez ao dia e a desinfecção, com a utilização de Benzocreol ${ }^{\circledR}$ a $2 \%$, respeitou intervalos de 21 dias. Houve aplicação de vacina contra clostridioses, Excell $10^{\circledR}$, de forma, que os animais foram vacinados aos 30 e 60 dias de vida, recebendo reforço anual. A aplicação de vermífugos ocorreu em ovelhas 24 horas após a parição e em borregos aos 30 e 60 dias de vida. Nas demais categorias utilizou-se o conceito de vermifugação estratégica com a aplicação de método FAMACHAC, sendo vermifugados somente os animais que apresentaram sintomatologia como emagrecimento, baixa qualidade do pelo e mucosas hipocoradas. Foi utilizado manejo preventivo contra coccidiose, sendo feito o uso de coccidiostático em animais com 30 e 60 dias de nascimento e animais com sintomatologia clínica de eimeriose.

A seleção das matrizes para a aplicação do protocolo hormonal foi realizada através de histórico, avaliação clínica e ultrassonografia do sistema reprodutivo, realizada com o aparelho da Pie Medical Aquila ${ }^{\circledR}$, por via transretal e frequência de $6 \mathrm{MHz}$. Foram submetidas a seleção 27 matrizes sendo afastadas 8 fêmeas por motivo de prenhez e 1 fêmea com imagem ultrassonográfica sugestiva de reabsorção embrionária. Desta forma, foram utilizadas 17 ovelhas, que fazem parte do registro de genealogia da Associação Brasileira de Criadores de Ovinos (ARCO), pluríparas com idade média de 3,4 anos (ovelha mais jovem: 2 anos e 7 meses; ovelha mais velha: 5 anos) e peso médio de $60 \mathrm{~kg}$.

Avaliaram-se dois protocolos distintos. O protocolo A1, obteve duração de cinco dias de forma que no dia 0 foi administrada P4-300 - Progesterona na dose de 1,0 ml (300 mg) por animal e no dia 5 foi aplicado Novormon (ECG) na dose de 1,4 ml (280 UI) e Ciosin (Cloprostenol Sódico) na dose de - 0,6 $\mathrm{ml}(0,150 \mathrm{mg})$ por animal. $\mathrm{O}$ protocolo A2, durou seis dias, sendo no dia 0 administrado P4-300 Progesterona na dose de 1,0 ml e no dia 3 foi aplicada a segunda dose de P4-300 - Progesterona na dose de 1,0 ml por animal; no dia 5 foi aplicado Novormon (ECG) na dose de 1,4 $\mathrm{ml}$ e Ciosin (Cloprostenol Sódico) na dose de - 0,6 ml para cada animal. Dessa forma, caracterizando protocolo curto de sincronização hormonal(Takada et al., 2009).

Para detecção de cio foram utilizados rufiões, preparados por procedimento mecânico de colocação de pano de algodão, cobrindo a região prepucial do macho e fixado por cordas na região dorsal, que impedia a cópula durante a rufiação. Eles eram postos em contato com as fêmeas, a partir de 24 horas após a última aplicação de PGf2 $\alpha$ e eCG, respeitando o intervalo de 2 em 2 horas, perfazendo um total de 72hs de observação. O diagnóstico de prenhez foi feito por meio de exame ultrassonográfico.

As respostas em relação ao efeito da progesterona no cio foram analisadas pelo teste de MannWhitney de significância $(\mathrm{P}>0,05)$, já o efeito do protocolo na prenhez foi submetido ao teste quiquadrado de significância $(\mathrm{P}<0,05)$.

\section{Resultados e discussão}

A indução ocorre quando há utilização de terapia hormonal que acarreta ocorrência de cio e ovocitação (Hafez \& Hafez, 2004). A sincronização do estro é obtida quando acontece a concentração 
de fêmeas nessa fase do ciclo em um intervalo de 24 a 72 horas (Balaro et al., 2017). Considerando que a apresentação de cio ocorreu em média 4,08 meses após o primeiro protocolo, sendo a variação de 1 mês até 6 meses e 14 dias, não se verificou indução e sincronização estral. Diante das não ocorrências de tais eventos foram consideradas como variáveis os dados relacionados à apresentação de cio e porcentagem de partos, sendo comparados os índices das ovelhas que foram submetidas ao tratamento hormonal e das que não estiveram inseridas no protocolo.

Simonetti et al. (1999) descreveram a distribuição da ocorrência de estro em ovelhas submetidas a utilização de protocolo com esponjas intravaginais impregnadas de Medroxiprogesterona (MAP), em seus resultados, $92,93 \%$ das matrizes apresentaram cio em menos de 72 horas. Nos estudos de Castilho et al. (2013), também em relação à utilização de implantes de MAP na sincronização de fêmeas ovinas, os dispositivos foram mantidos por 9 ou 14 dias, apresentando índices de sincronização de $61 \%$ e $80 \%$, respectivamente. Já no experimento de Morotti et al.(2013) em que um grupo de vacas Nelore foi submetido à utilização progesterona injetável, com o intuito de avaliação da dinâmica folicular, foi demonstrado que essa ocorreu de forma similar, mas os níveis de ovocitação foram menores em relação às fêmeas em que foram colocados os implantes intravaginais. Nessa pesquisa, apenas $37,5 \%$ das matrizes bovinas passaram pelo processo ovocitatório num período de observação que foi feito até 48 horas, enquanto o grupo que utilizou pessários obteve índice de $90 \%$. Segundo o autor, as peculiaridades do metabolismo da progesterona, quando administrada por via injetável, podem interferir na dinâmica folicular resultando em níveis variados de LH e maior período de crescimento do folículo dominante. Verificou-se que a utilização da progesterona injetável no protocolo de sincronização em ovelhas foi congruente com testes aplicados a matrizes bovinas de forma que quando comparados com os estudos que dispuseram de implantes, não levou a resultados satisfatórios de sincronização.

Para avaliação dos índices de cio obtidos no rebanho, durante o ano de 2018, foram consideradas as matrizes que participaram do protocolo e as que não passaram pela aplicação hormonal. Na comparação entre os dois grupos citados, não foram encontradas diferença significativa $(\mathrm{P}>0,05)$. Özyurtlu et al.(2011) avaliaram métodos de indução de ciclo estral em ovelhas por meio da utilização de esponjas com diferentes períodos de retirada, sendo que nos grupos que fizeram a utilização do implante, os índices de apresentação de estro, variaram entre $91,6 \%$ a $100 \%$; enquanto o grupo controle obteve um índice de $25 \%$, demonstrando assim grande diferença percentual entre os grupos submetidos e o grupo não submetido ao tratamento. Os índices de parição apresentados nas matrizes submetidas ao protocolo em 2018 foram de 43,75\%, já no grupo de ovelhas que não foi sujeitado a interferência hormonal exógena, o índice foi de $56,25 \%$.

Verificou-se efeito negativo significativo no parto de ovelhas submetidas ao protocolo $(\mathrm{p}<0,05)$. Em comparação, Fleisch et al. (2013) obtiveram resultados sobre a eficiência de dispositivos intravaginais impregnados com progesterona em ovelhas Lancaune com diferentes períodos de retirada, no grupo submetido ao protocolo curto de indução e sincronização, obtiveram um resultado de parição de $71,7 \%$. Santos \& Barcelos (2012),também realizaram estudos com implantes em ovinos, contudo efetuaram um protocolo com progestágenos de implantação auricular, associado a aplicação de eCG e PGF $2 \alpha$ em ovelhas advindas de cruzamentos Texel x Ilê de France, sendo alcançado um índice de parição de 93,0\%. Assim, o resultado do índice de partos em matrizes ovinas submetidas a aplicação de progesterona injetável apresentado nesse artigo, quando comparado aos diferentes protocolos de sincronização com utilização de implantes mostram-se inferiores, demonstrando que levam a um decréscimo na produção de borregos.

\section{Conclusão}

O protocolo com utilização de progesterona exógena injetável não acarretou indução e sincronização do cio. $\mathrm{Na}$ análise comparativa entre as matrizes que foram submetidas ao tratamento e as que não estavam inseridas no protocolo hormonal, não houve interferência significativa na porcentagem de apresentação de cio anual. Em relação ao índice de partos, houve influência negativa do tratamento, sendo demonstrado um índice menor de partos anuais nas ovelhas pertencentes ao grupo de aplicação hormonal. Assim, esse estudo proporcionou bases para outras investigações relacionadas a novas formas de aplicação de progesterona em protocolos de indução e sincronização estral em ovinos. 


\section{Referências bibliográficas}

ANUALPEC. (2018). Anuário da Pecuária Brasileira (20th ed. Vol. 1). São Paulo, São Paulo, Brasil: Instituto FNP.

Balaro, M. F. A., Souza, J. M. G. \& Cortês, L. R. (2017). Sincronização e indução do estro em caprinos leiteiros. Revista Brasileira de Reprodução Animal,41330-339.

Castilho, C., Almeida, M. F., Costa, M. Z., Cesare, Â. G. \& Gabriel Filho, L. R. A. (2013). Protocolos de indução e sincronização do estro em ovelhas. Ciência Animal Brasileira,14(1):91-97.

Colazo, M. G. \& Mapletoft, R. (2017). Fisiología del ciclo estral bovino. Ciencia Veterinaria,16(2):3146.

Fleisch, A., Piechotta, M., Bollwein, H. \& Janett, F. (2013). Fertility after treatment with Eazi-breedTM CIDR $\mathrm{G}$ for 6 or 12 days outside the breeding season in Lacaune dairy sheep. Schweizer Archiv fur Tierheilkunde, 155(7):391-398.

Hafez, B. \& Hafez, E. (2004). Reprodução Animal (Vol. 1, pp. 513): Manole: São Paulo, Brasil.

Köppen, W. \& Geiger, R. (1928). Klimate der Erde. Gotha: Verlag Justus Perthes. Wall-map 150cmx200cm. Lehninger, N. D. L. (2006). Principios de bioquímica. São Paulo.

Morotti, F., Campos, J. T., Oliveira, E. R. \& Seneda, M. M. (2013). Ovarian follicular dynamics of Nelore (Bos indicus) cows subjected to a fixed-time artificial insemination protocol with injectable progesterone. Semina: Ciências Agrárias,34(2):3859-3866.

Özyurtlu, N., Ay, S. S., Küçükaslan, İ. \& Güngör, Ö. (2011). Effect of subsequent two short-term, short-term, and long-term progestagen treatments on fertility of Awassi ewes out of the breeding season. Ankara Üniversitesi Veteriner Fakültesi Dergisi,58(2):105-109.

Reece, W. O. \& Penteado Júnior, N. (1996). Fisiologia de animais domésticos. São Paulo: Roca.

Santos, F. C. C. \& Barcelos, R. A. D. (2012). Eficiência de protocolos de sincronização de estro em ovelhas. Revista Brasileira de Reprodução Animal,36(3):202-205.

Simonetti, L., Ramos, G. \& Gardón, J. C. (1999). Estrus presentation and distribution in ewes treated with intravaginal sponges impregnated with medroxyprogesterone acetate (MAP) in combination with pregnant mare serum gonadotropin (PMSG). Brazilian Journal of Veterinary Research and Animal Science,36(5):1-4.

Sousa, R. T., Gonçalves, J. L., Fonteles, N. L. O., Santos, C. M., Ricci, G. D., Albuquerque, F. H. M. A. R. \& Bomfim, M. A. D. (2015). Características reprodutivas de ovelhas Morada Nova e Somalis Brasileira. PUBVET,9(11):467-501.

Souza, M. I. L. (2013). Indução e sincronização de estro em ovelhas: desafios e potencial. Revista Brasileira de Reprodução Animal, Belo Horizonte,37(2):220-225.

Takada, L., Bicudo, S. D., Rodrigues, C. F. C., Coelho, L. A. \& Perri, S. H. V. (2009). Sincronização do estro e da ovulação utilizando protocolos de curta duração durante a pré-estação reprodutiva em ovelhas Suffolk. Acta Scientiarum. Animal Sciences,31(4):453-460.

Uribe-Velásquez, L. F., Oba, E. \& Souza, M. I. L. (2008). Población folicular y concentraciones plasmáticas de progesterona (P4) en ovejas sometidas a diferentes protocolos de sincronización. Archivos de Medicina Veterinaria,40(1):83-88.

Uribe-Velásquez, L. F., Souza, M. I. L. \& Osorio, J. H. (2010). Resposta ovariana de cabras submetidas a implantes de progesterona seguidos de aplicações de gonadotrofina coriônica equina. Revista Brasileira de Zootecnia,39(6):1214-1222.

Recebido: 6 de junho, 2019.

Aprovado: 5 de julho, 2019.

Publicado: 5 de setembro, 2019.

Licenciamento: Este artigo é publicado na modalidade Acesso Aberto sob a licença Creative Commons Atribuição 4.0 (CC-BY 4.0), a qual permite uso irrestrito, distribuição, reprodução em qualquer meio, desde que o autor e a fonte sejam devidamente creditados. 